\title{
Recent results from the Tevatron
}

\author{
Costas Vellidis ${ }^{1, a}$, on behalf of the CDF and DZero Collaborations \\ ${ }^{1}$ Fermi National Accelerator Laboratoty, Batavia, IL 60510, USA
}

\begin{abstract}
The Tevatron $p \bar{p}$ collider was shut down in 2011, after 10 years of high performance operation at a center-of-mass energy $\sqrt{s}=1.96 \mathrm{TeV}$ in Run II. The two experiments, CDF and DZero, continue to analyze the collected data, aiming to extract all possible information regarding studies of the standard model and searches for new physics. A short review of some of the recent measurements at the Tevatron, and of the impact of the Tevatron program to high energy physics, is presented.
\end{abstract}

\section{Introduction}

The Tevatron proton-antiproton collider at the Fermi National Accelerator Laboratory (Fermilab) near Chicago, USA, was shut down on September 30, 2011, after ten years of operations at a collision energy of $1.96 \mathrm{TeV}$ during Run II. The collider delivered $12 \mathrm{fb}^{-1}$ of integrated luminosity and each experiment, CDF and DZero, acquired data corresponding to $10 \mathrm{fb}^{-1}$. These samples are now intensively analyzed by the CDF and DZero Collaborations, currently consisting of about 400 active members each, coming from about 60 (CDF) and 70 (DZero) institutions from across the World. The samples are well understood and, based on the mature analysis knowledge developed by both Collaborations, they provide an enormous potential for physics within and beyond the standard model. Up-to-date listings of the Tevatron Run II results can be found on the Web at the public pages of physics results of the two experiments [1].

\section{Higgs boson measurements}

Both Tevatron experiments have completed their program of searches for the standard model (SM) Higgs boson in all decay channels, using the full luminosity in all channels [2]. The top left window of Fig. 1 shows the combined exclusion limits of the searches from both experiments in all channels at the $95 \%$ confidence level (CL) as a function of the Higgs boson mass hypothesis. The dashed line shows the expected limit assuming no Higgs boson (null hypothesis) with the corresponding oneand two-standard deviation (st.d.) uncertainty bands. The solid line shows the observed limit. The Higgs boson is excluded between 150 and $180 \mathrm{GeV} / \mathrm{c}^{2}$, mainly by the $\mathrm{H} \rightarrow \mathrm{WW}$ channel. There is an observed excess over the expected limit between for the null hypothesis between 115 and 150 $\mathrm{GeV} / c^{2}$, arising mainly from the $\mathrm{H} \rightarrow b \bar{b}$ channel. The significance of the excess at $125 \mathrm{GeV} / c^{2}$ is 3.0 st.d. The dash-dotted line shows the expected limit assuming a Higgs boson with a mass of 125 $\mathrm{GeV} / \mathrm{c}^{2}$ (test hypothesis). The observed limit shows a clear preference for the test hypothesis against the null hypothesis.

\footnotetext{
a e-mail: vellidis@fnal.gov
} 

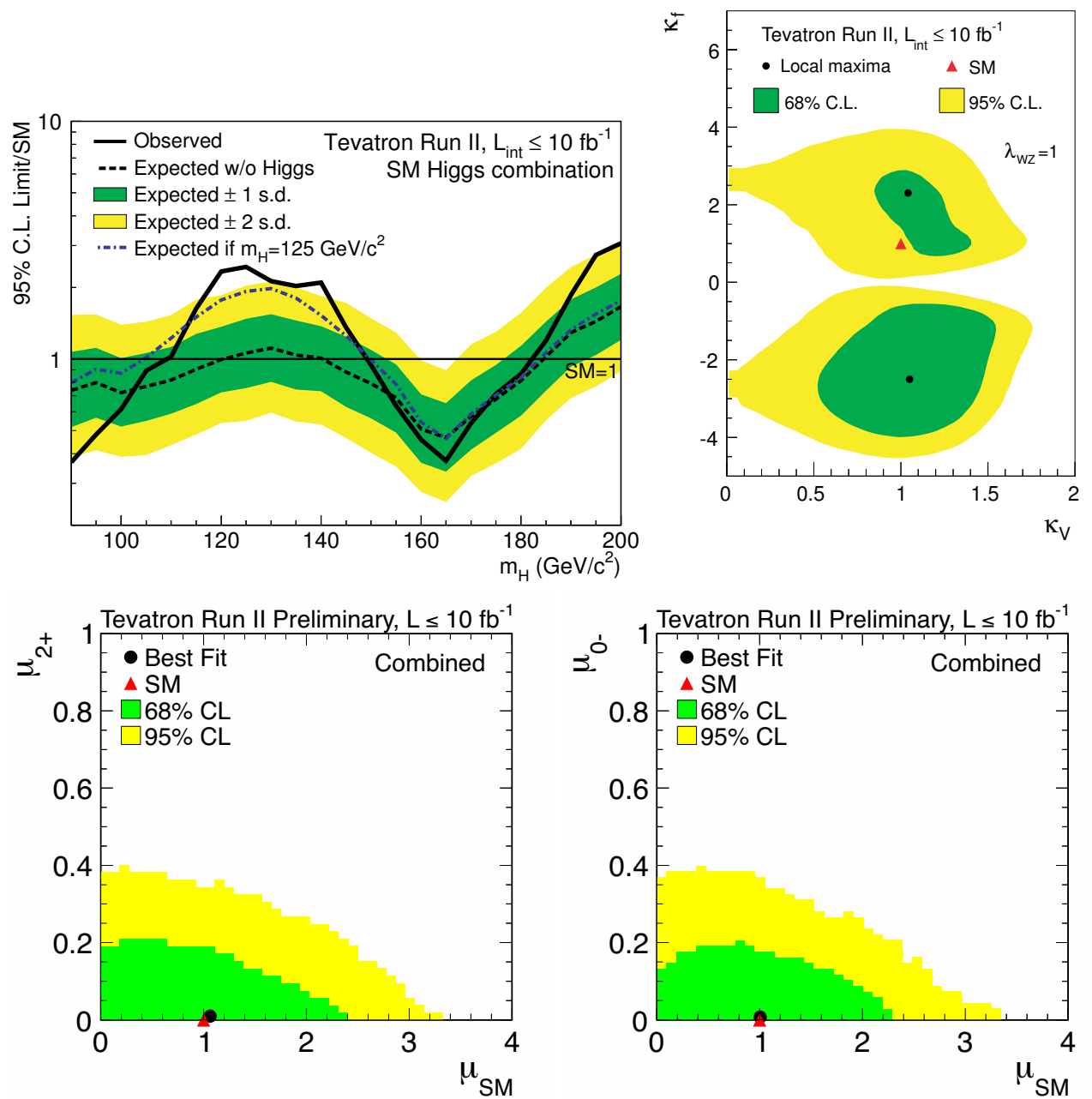

Figure 1. Top: Exclusion limits (left) and couplings of the SM Higgs boson at the Tevatron. Bottom: Tests of the spin and parity state of the Higgs boson at the Tevatron.

The couplings of the SM Higgs boson to the weak bosons and to fermions were derived from the cross sections times branching ratios (BR) measured in all decay channels by both experiments, accounting for all production modes [2]. The top right window of Fig. 1 shows the fermionic $\left(\kappa_{f}\right)$ and bosonic $\left(\kappa_{V}\right)$ couplings $(\kappa=1$ means SM coupling) derived from the measurements, with the corresponding one- and two-st.d. uncertainty regions. The result is consistent with the SM prediction. The sign degeneracy arises from the quadratic dependence of the cross sections and BRs on the couplings.

In more recent studies, the spin and parity state of the Higgs boson has been tested by both experiments in the $\mathrm{H} \rightarrow b \bar{b}$ channel against the SM hypothesis of a $0^{+}$state [3]. The bottom windows of Fig. 1 show the couplings ( $\mu=1$ means SM coupling for a $0^{+}$state) for a pseudoscalar $\left(0^{-}\right)$state hypothesis (left) and a graviton-like $\left(2^{+}\right)$state hypothesis (right) assuming the presence of a SM Higgs boson $\left(0^{+}\right.$ 
state) in both cases, with the corresponding one- and two-st.d. uncertainty regions. The couplings are derived from multivariate discriminants using kinematic variables characteristic of the spin and parity state, such as the total invariant mass of the $\mathrm{V} b \bar{b}$ final state ( $\mathrm{V}=\mathrm{W}$ or $\mathrm{Z}$ boson). Consistency with the SM is observed.

\section{Searches for new physics}

The left window of Fig. 2 shows the expected exclusion limit from the search for a $Z^{\prime} \rightarrow t \bar{t}$ decaying boson at $\mathrm{CDF}$ [4], as a function of the $\mathrm{Z}^{\prime}$ boson mass, compared with corresponding limits from other experiments. The CDF limit is derived from $t \bar{t} \rightarrow$ lepton+jets events and it is currently the World best up to a mass of $700 \mathrm{GeV} / c^{2}$. The right window of Fig. 2 shows the expected exclusion limit from the search for a $\mathrm{W}^{\prime} \rightarrow t \bar{b}$ decaying boson at CDF [5], as a function of the $\mathrm{W}^{\prime}$ boson mass, compared with corresponding limits from other experiments. The CDF limit is derived from $t \bar{b} \rightarrow \mathbb{H}_{T} b \bar{b}$ events $\left(\mathbb{E}_{T}\right.$ is the missing transverse energy) and it is currently the World best up to a mass of $700 \mathrm{GeV} / c^{2}$.
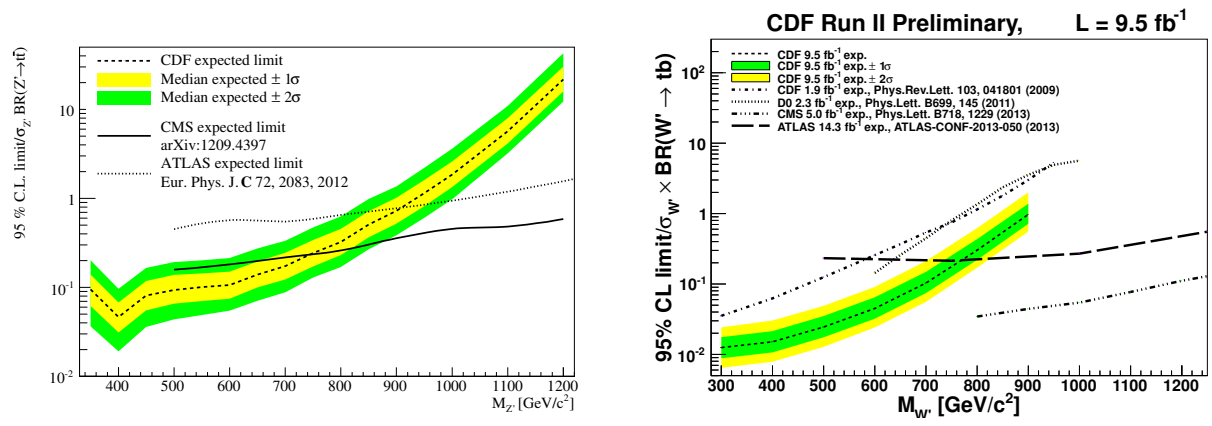

Figure 2. Limits on exotic weak boson production from CDF.

\section{Top quark measurements}

The electroweak mode of top quark production (through the exchange of a virtual $\mathrm{W}$ boson between an initial-state pair of light quarks and a final-state $t b$ pair), known as "single top production", was first observed by CDF and DZero in 2009 [6] by measuring the combined cross section for single top production in the s-channel (where the virtual $\mathrm{W}$ boson is time-like) and the t-channel (where $\mathrm{W}$ is space-like). This observation verified the SM prediction for that process. Recently, the two experiments measured the cross section for s-channel single top production using their full datasets and both reported evidence for this sub-process, with an observed significance of 4.2 st.d. for CDF [7] and 3.8 st.d. for DZero [8]. DZero measured the cross section in the $t \bar{b} \rightarrow$ lepton $+b \bar{b}$ topology, whereas CDF measured the cross section in both the $t \bar{b} \rightarrow$ lepton $+b \bar{b}$ and the $t \bar{b} \rightarrow \mathbb{E}_{T}+b \bar{b}$ topologies and combined the two results. A top quark mass of $172.5 \mathrm{GeV} / c^{2}$ was assumed in all measurements. The two experiments combined their results to obtain the first observation of s-channel single top production [9], with an expected siginficance of 5.1 st.d. and an observed significance of 6.3 st.d. The left window of Fig. 3 shows the distribution of the signal-to-background ratio logarithm, which is used as the final discriminant and manifests the sensitivity of the single top signal in the signal-rich bins. The right window of Fig. 3 shows the probability density of the log-likelihood ratio for the null (background only) and test 

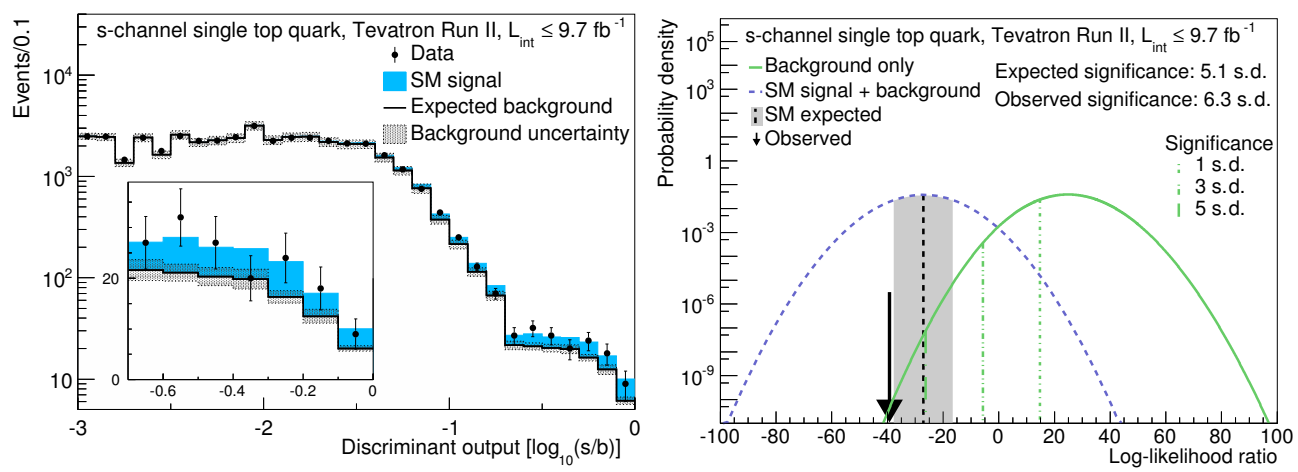

Figure 3. S-channel single top quark observation at the Tevatron. The discriminant (left) and log-likelihood ratio (right) are shown. The inset plot in the left window shows the highest $\mathrm{s} / \mathrm{b}$ bins, which are the most sensitive to the s-channel single top signal.

(SM signal plus background) hypotheses, derived from pseudo-experiments, from which the expected and observed significance is measured.

A subtlety observed in the Tevatron data concerns the forward-backward asymmetry in the production of top quark pairs, i.e. how many top quarks are produced closer to the direction of the initial-state protons vs. how many anti-top quarks are produced closer to the direction of the initial state anti-protons. This asymmetry depends on the initial state, which is CP-invariant at the Tevatron, and it originates from the interference of the leading-order (LO) $t \bar{t}$ production mechanisms with nextto-leading-order (NLO) mechanisms, but also with possible mechanisms beyond the standard model (BSM). Fig. 4 on the top left shows the normalized cross section for $t \bar{t}$ production differential in the top quark angle about the initial-state proton direction in the $t \bar{t}$ rest frame, measured at CDF from $t \bar{t} \rightarrow$ lepton+jets events [10] and compared with the NLO SM prediction. The measured cross section is clearly asymmetric, with an asymmetry stronger than the prediction. CDF analyzed the asymmetry in terms of Legendre moments and found that only the first moment deviates significantly from the NLO SM prediction, as shown on the top right window of Fig. 4. More recently, DZero also measured the asymmetry from $t \bar{t} \rightarrow$ lepton+jets events as a function of the $t \bar{t}$ invariant mass [11]. The results are shown in the bottom left window of Fig. 4, compared with the CDF results [12] and the NLO SM prediction. The current status of the inclusive asymmetry measurements using the full Tevatron luminosity and the NLO SM prediction is summarized in the bottom right window of Fig. 4 [13]. With the new DZero measurements, the tension with the NLO SM prediction is reduced and the average of the measurements shows a moderate agreement with the prediction.

CDF recently extended the studies of forward-backward production asymmetries to the bottom quark sector by using doubly $b$-tagged dijet events [14]. Fig. 5 on the left shows the measured asymmetry as a function of the $b \bar{b}$ invariant mass, compared with the predictions from NLO SM and from two axi-gluon models with different axi-gluon mass. On the right, the Figure shows the probability density for the asymmetry, derived from pseudo-experiments, with the one- and two-st.d. uncertainty regions. The measurements exclude the low-mass axi-gluon model.

One of the primary goals of the Tevatron Run II physics program has been the precise measurement of the top quark mass. CDF recently updated the measurements of the top mass in the all-jets [15] and dilepton [16] decay modes of $t \bar{t}$ events using the full luminosity and the template method to extract the mass from the data. In this method, the top mass distribution is reconstructed from the $t \bar{t}$ 

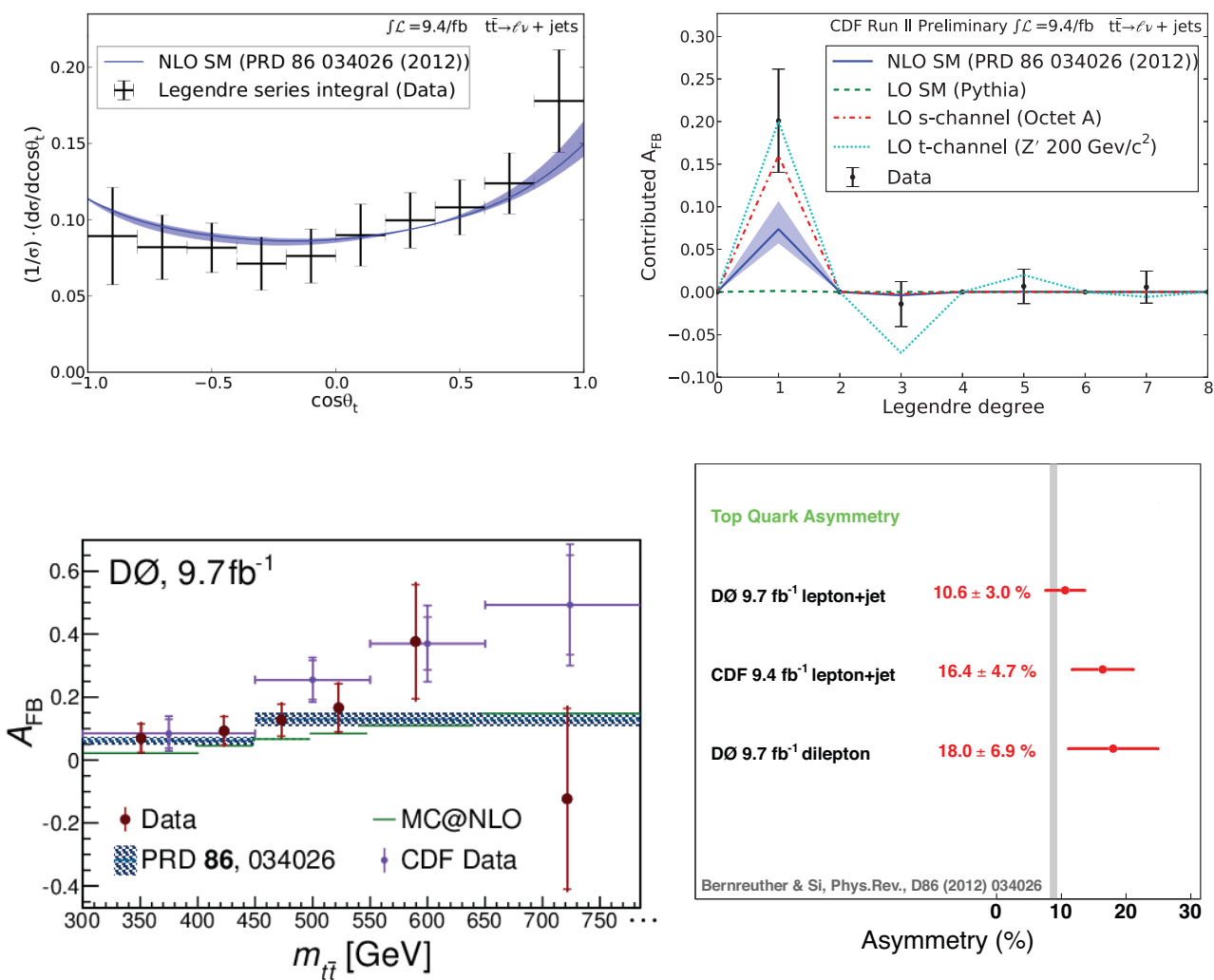

Figure 4. Studies of the top pair forward-backward asymmetry at the Tevatron. Top: Top pair angular distribution (left) and its analysis in Legendre moments (right). Bottom: Differential (left) and inclusive (right) asymmetry results.

events simulated with different input top mass values (making some assumptions for the undetected neutrinos in the dilepton case) and from simulated background events, and then it is fit to the experimental distribution reconstructed from the $t \bar{t}$ candidate events in the data. In the all-jets measurement, the reconstructed mass of the hadronically decaying $\mathrm{W}$ bosons that originate from the top quark decays is used to constrain the energy scale of jets originating from light quarks, which is typically the source of largest systematic uncertainty in top mass measurements. This technique, called "in situ" jet energy scale (JES) calibration, is not possible in the dilepton measurement, where the $\mathrm{W}$ bosons decay leptonically and the undetected neutrinos from their decays do not allow for reconstruction of the $\mathrm{W}$ mass. Fig. 6 shows the fits for the all-jets (top left) and dilepton (top right) measurements, with the background-only and the signal+background templates, and the data points. The derived top mass values are $\left(175.07 \pm 1.19_{\text {stat }} \pm 1.55_{\text {syst }}\right) \mathrm{GeV} / c^{2}=(175.07 \pm 1.95) \mathrm{GeV} / c^{2}$ from the all-jets fit and $\left(170.80 \pm 1.83_{\text {stat }} \pm 2.69_{\text {syst }}\right) \mathrm{GeV} / c^{2}=(170.80 \pm 3.26) \mathrm{GeV} / c^{2}$ from the dilepton fit, both dominated by the total systematic uncertainty.

Recently, DZero updated the top mass measurement in the lepton+jets decay mode using the full luminosity and the matrix element method to extract the mass from the data [17]. In this method, the sensitivity of the SM cross section for $t \bar{t}$ production on the top mass is used to derive the mass by 

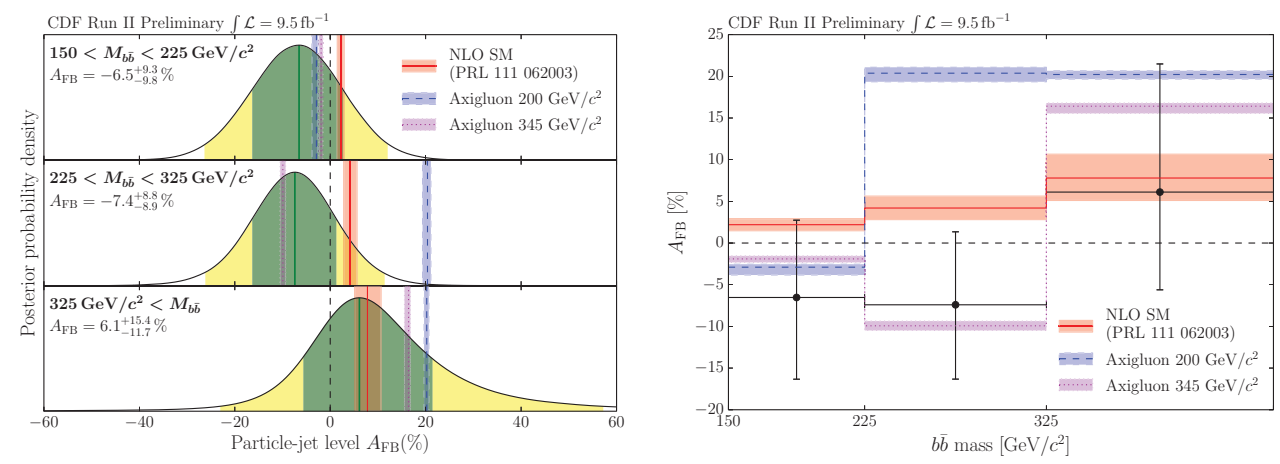

Figure 5. Measurement of the bottom pair forward-backward asymmetry at CDF, in two alternative representations of its dependence on the bottom pair invariant mass.

fitting the event yield for background and signal, as a function of the top mass, to the data. Like in the all-jets decay mode, the reconstructed mass from the hadronically decaying $\mathrm{W}$ boson originating from one top quark in the event is used for an "in situ" JES calibration. The bottom left window of Fig. 6 shows the two-dimensional fit of the top mass and JES with the one-, two- and three-st.d. contours. The result for the top mass is $\left(174.98 \pm 0.58_{\text {stat }+\mathrm{JES}} \pm 0.49_{\text {syst }}\right) \mathrm{GeV} / c^{2}=(174.98 \pm 0.76) \mathrm{GeV} / c^{2}$, which is currently the most precise single measurement of the top mass from the Tevatron. Fig. 6 in the bottom right shows the most recent Tevatron combination of top mass measurements that includes all of the above results [18]. All measurements are consistent within uncertainties. The combined result is $\left(174.34 \pm 0.37_{\text {stat }} \pm 0.52_{\text {syst }}\right) \mathrm{GeV} / c^{2}=(174.34 \pm 0.64) \mathrm{GeV} / c^{2}$, which has a total uncertainty of only $0.37 \%$.

\section{Measurements of standard model processes}

The Tevatron experiments continue their programs of high precision tests of SM predictions in the domains of quantum chromodynamics (QCD) and electroweak phenomena. Fig. 7 on the left shows the cross section for WW diboson production measured at CDF, inclusive, as a function of the jet multiplicity, and as a function of the jet transverse energy $E_{T}$ in the 1-jet bin [19]. On the right, the Figure shows the CDF measurement of the normalized cross section for $\mathrm{W}$ boson production associated with low momentum charm production (in the form of a $D^{*}$ meson) differential in the transverse momentum of the $D^{*}$ meson [20]. Both measurements use leptonic decays of the $\mathrm{W}$ bosons. In both cases, there is good agreement between the data and the SM predictions within the uncertainties.

Fig. 8 on the left shows angular distributions of photon+3jets events yields measured at DZero, which are sensitive to multiple interactions between quarks and gluons (collectively called partons) in $p \bar{p}$ collisions [21]. On the right, the Figure shows the ratio of the cross section for $\mathrm{Z}$ boson plus a charmed jet production to the cross section for $\mathrm{Z}$ boson plus a light flavor jet production, differential in the jet transverse momentum, also measured at DZero [22]. The agreement with SM predictions is good for the multi-parton interaction test, but not good for the $\mathrm{Z}+$ charm measurement.

\section{Heavy quark flavor measurements}

The Tevatron data have unique capabilities for measurements in the heavy quark flavor sector, thanks to the CP-invariant initial state and the variety of low-momentum track-based triggers that have been 

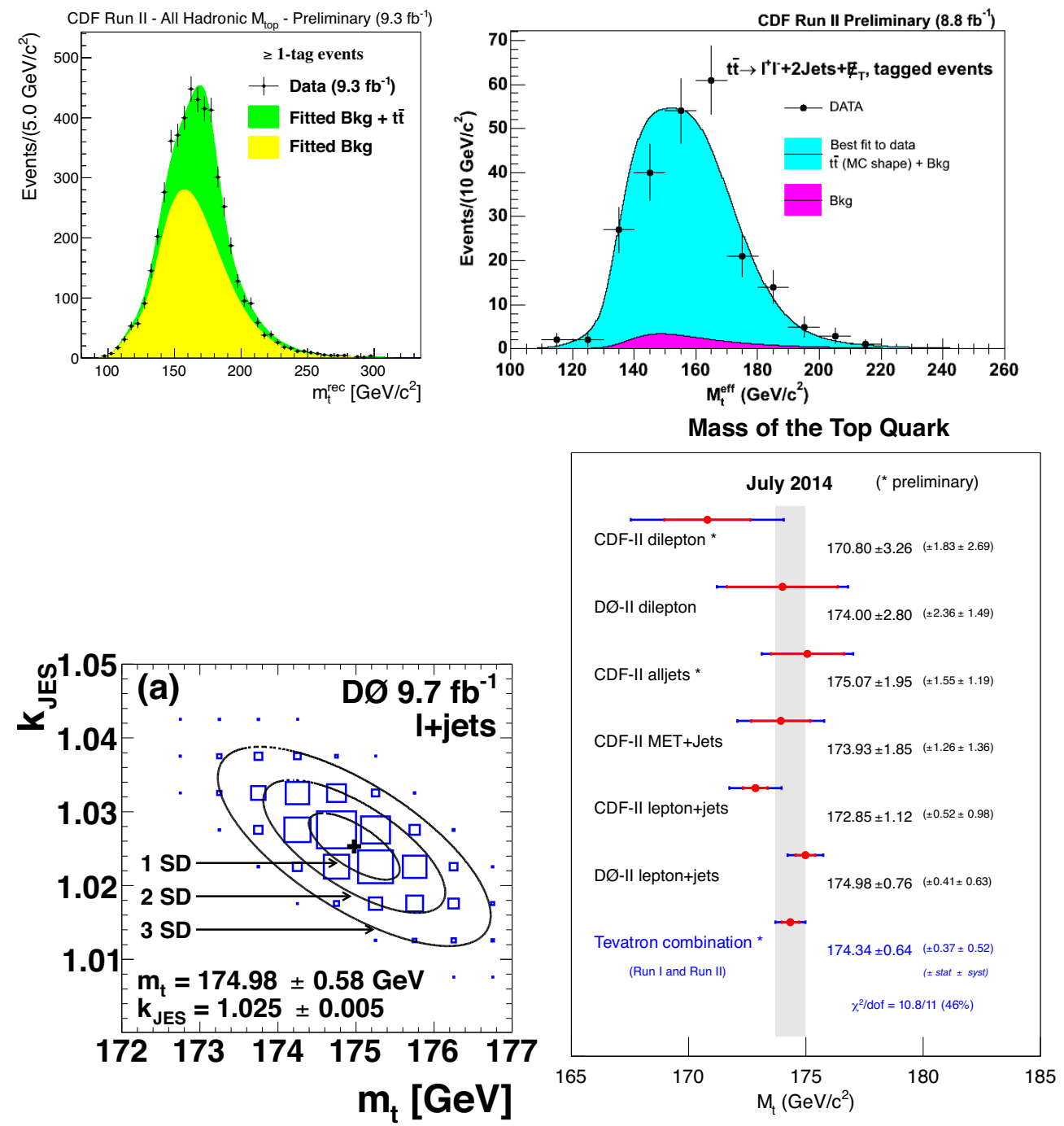

Figure 6. Top quark mass measurements at the Tevatron.

developed by both experiments. These features allow for an extensive physics program in the heavy flavor sector. Fig. 9 shows the Q-value spectrum of $B \pi$ pairs from excited $B$ meson states in the neutral (left) and charged (right) final states reconstructed from CDF data [23]. In both cases, a newly observed structure is visible between 0.5 and $0.6 \mathrm{GeV} / c^{2}$. This is identified as the signature of a $B(5970)$ state that can be interpreted as a radial excitation of either a $B_{1}$ meson state decaying into a $B^{*} \pi$ pair (where a soft photon from the subsequent $B^{*}$ decay is missing detection) or a $B_{0}^{*}$ or a $B_{2}^{*}$ state decaying into a $B \pi$ pair. This measurement provides the first evidence for the $B(5970)$ state.

DZero looked for a CP-violation asymmetry $\left(\mathrm{A}_{\mathrm{CP}}\right)$ in $B^{ \pm} \rightarrow J / \psi K^{ \pm} \rightarrow \mu^{+} \mu^{-} K^{ \pm}$and $B^{ \pm} \rightarrow$ $J / \psi \pi^{ \pm} \rightarrow \mu^{+} \mu^{-} \pi^{ \pm}$decays [24]. Fig. 10 on the left shows the invariant mass distribution of the muon- 

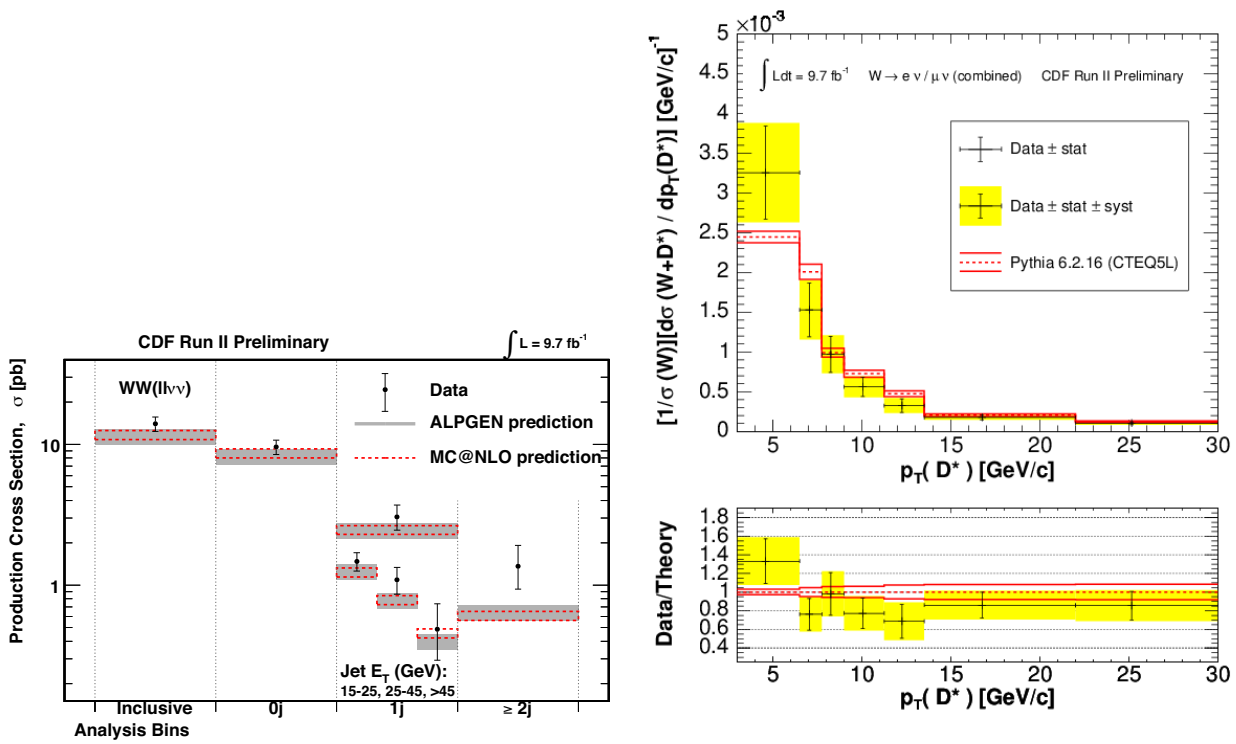

Figure 7. Cross section measurements of standard model processes involving W boson production at CDF.

pair plus kaon or pion system, from which the $B^{ \pm}$meson is reconstructed, and on the right it shows the yield difference between the two charge states, from which the asymmetry is extracted. An $\mathrm{A}_{\mathrm{CP}}$ of $(0.59 \pm 0.36) \%$ for the $J / \psi K$ system and of $(-4.2 \pm 4.8) \%$ for the $J / \psi \pi$ system is measured. Despite the relatively large uncertainties, these results are the most accurate $A_{C P}$ measurements in leptonic channels to-date, owed to the ability of the DZero detector to regularly reverse the magnetic field of the spectrometer, which cancels out detector asymmetries.

\section{Future plans and data preservation}

The plan of the Tevatron experiments for the future is to finish the physics program with the full luminosity, focusing on measurements where the Tevatron data are still competitive with the LHC data. Such measurements involve cases where the backgrounds are substantially lower or very lowmomentum triggers are required or a CP-invariant initial state plays an important role. The program also involves the completion and publication of final Tevatron combinations. To complete the physics program, a joint effort by Fermilab and the Tevatron experiments is ongoing, aiming to preserve the data, analysis tools, and analysis knowledge in the framework of a Long Term Data Preservation project. These include nearly 10 petabytes of data for each experiment and about 18,000 analysis notes in total. This project is now close to completion and will maintain the ability of analyzing the unique $p \bar{p}$ data samples from the Tevatron experiments in the long term future.

\section{The Tevatron legacy}

The Tevatron was leading high energy physics for two decades, 1990-2010, contributing to all relevant areas in science and technology. In physics, it was the place where the top quark, the heaviest known 

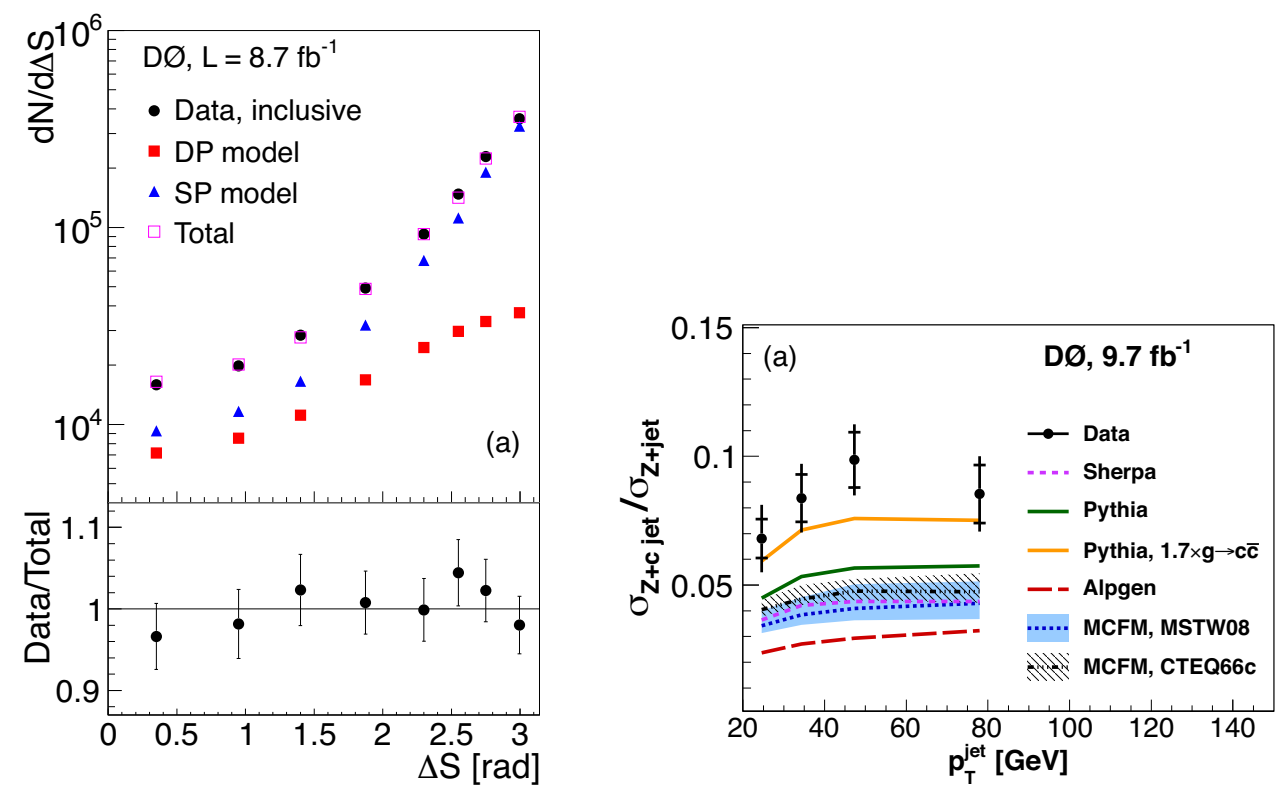

Figure 8. Studies of multi-parton interactions in $p \bar{p}$ collisions at DZero, in photon +3 jets production (left) and in $\mathrm{Z}+$ charm production (right).
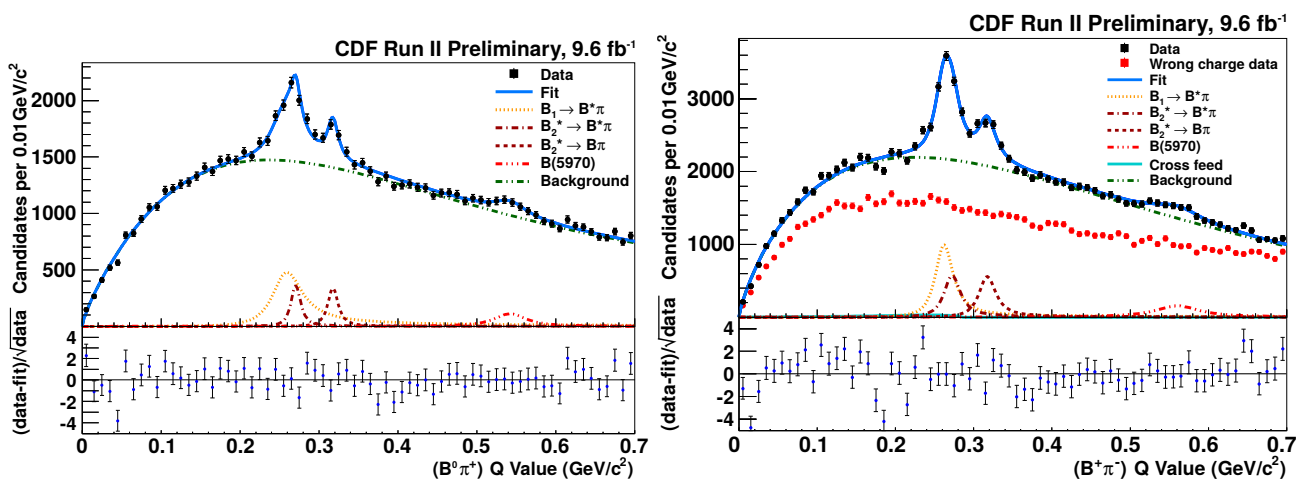

Figure 9. New B state observed at $\mathrm{CDF}$ at a Q-value near $0.6 \mathrm{GeV} / c^{2}$. The two peaks around $0.3 \mathrm{GeV} / c^{2}$ arise from known states.

particle, was discovered and its properties were measured for the first time. The Tevatron provided the first evidence for the standard model Higgs boson in fermionic final states. Precision measurements in the electroweak and QCD areas were performed, including measurements of jet, photon, weak boson, and diboson production cross sections. Perhaps the most important measurement regarding precision is that of the $\mathrm{W}$ boson mass - the most precise measurement of this fundamental parameter of the SM to date. Substantial progress was made in the physics of heavy flavor states and mixing, while several cases of subtle behavior have been identified and resolved, such as CP violation in 

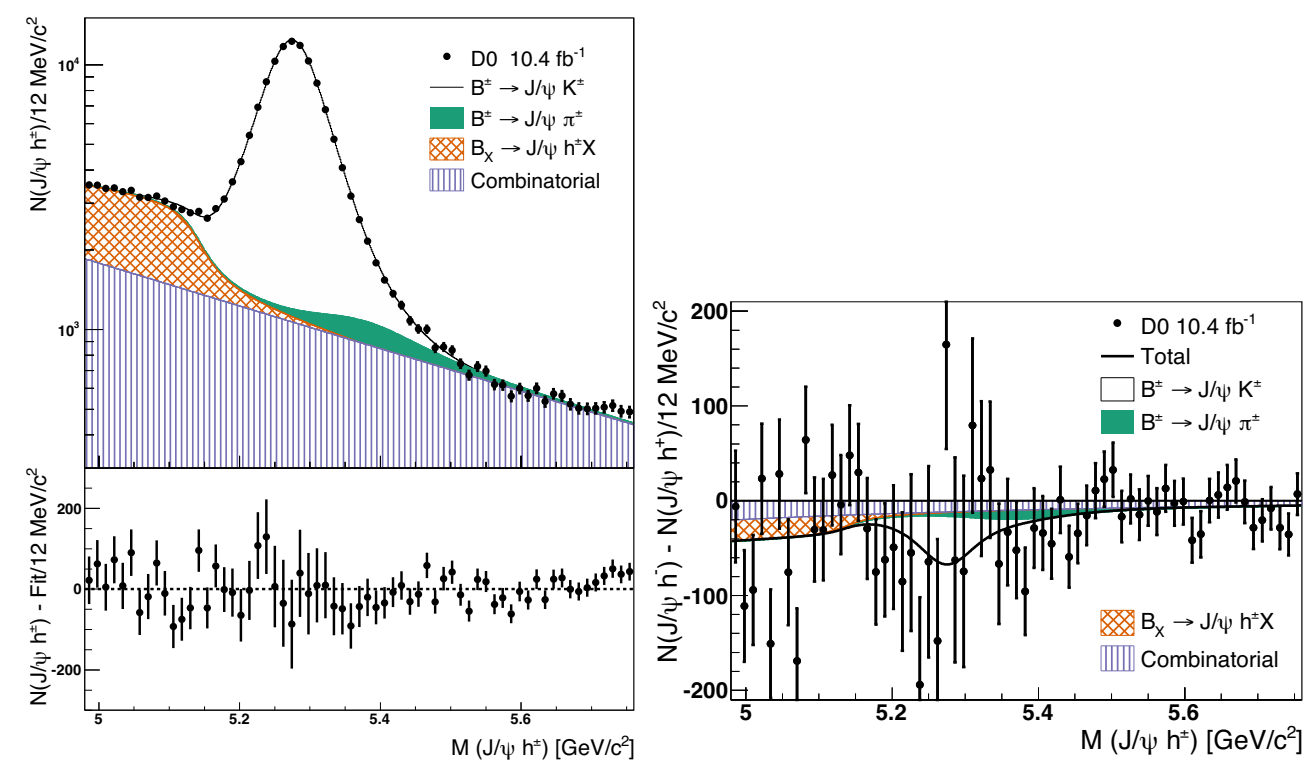

Figure 10. Measurement of CP violation asymmetries in $B$-meson decays at DZero.

heavy-flavored hadrons and the forward-backward asymmetry in top pair production. The volume of production of physics results from the Tevatron is represented by the number of publications per year in peer-reviewed journals, which is shown in the top two windows of Fig. 11. The total number of such publications from both experiments exceeds 1,100 over the last 25 years.

In hardware developments, tracking and collision vertex reconstruction using silicon detector were taken to a whole new level. Heavy flavor tagging was widely developed and used. The entire trigger concept in a hadron collider environment was reconsidered and advanced, including the secondary vertex trigger (SVT) and out-of-time triggers for the detection of long-living or slow particles. In the domain of data analysis, the importance of multivariate techniques was established in cases such as the search for the SM Higgs boson in the $b \bar{b}$ final state and the observation of single top quark production, where the signal is tiny relative to the irreducible background and does not have a clear resonance signature in a reconstructed invariant mass spectrum. Innovative experimental methods were first applied, such as the "in situ" JES calibration in top mass measurements.

In accelerator technology, major advances were the anti-proton recycler, the anti-proton cooling technique using electrons, as well as many innovations in cryogenic technology. The Tevatron was the first installation of mass-produced super-conducting magnets on an industrial scale.

A major part of the Tevatron legacy is the impact on the LHC, which took over the lead in high energy physics after 2010. The Tevatron was the place where it was first demonstrated that a wealth of precision measurements can be made at hadron colliders. Advanced analysis techniques were brought to full maturity, allowing for tiny signals to be extracted from huge (often complex mixtures of) backgrounds. The Tevatron proved that a huge gain in sensitivity can be achieved with data, painstaking experimental work, and a lot of ingenuity. Very important was the transfer of a huge amount of knowledge and experience through the Tevatron accelerator experts and physicists from the Tevatron experiments joining the LHC experiments. 

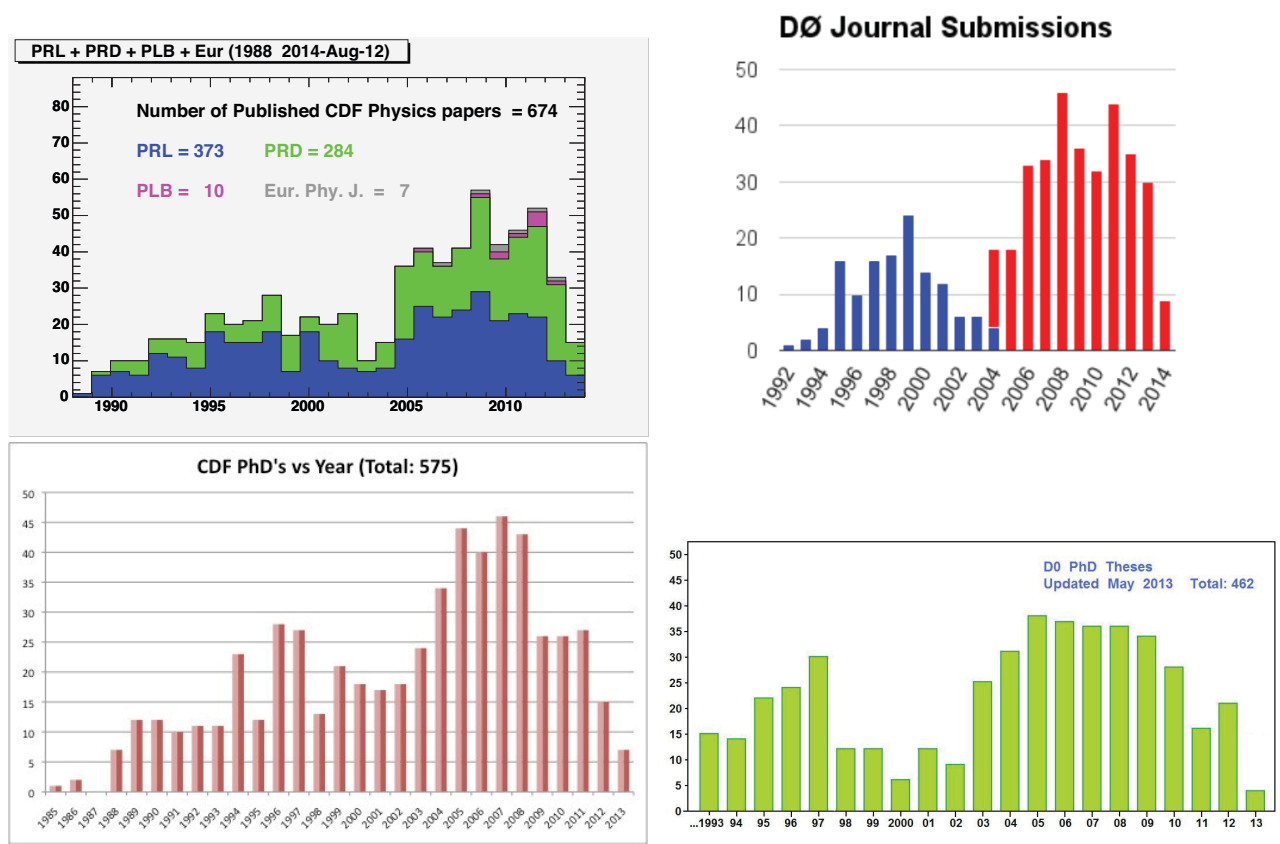

Figure 11. The Tevatron legacy in numbers.

The most important part of the Tevatron legacy was the formation of a new generation of scientists in physics and in the society at large, through the graduation of more than one thousand PhD students in 25 years (1988-2013), whose work was based on either of the two Tevatron experiments, as shown in the bottom two windows of Fig. 11.

\section{Summary}

The Tevatron experiments, CDF and DZero, three years after the cease of their operations, keep producing high-quality physics results, with emphasis to precision and to measurements that are difficult to conduct at the LHC. A huge legacy in science and technology is inherited to the high-energy physics community after 25 years of research and development. This legacy is represented by more than 1,100 publications in peer-reviewed journals and more than 1,000 $\mathrm{PhD}$ theses from both experimements. The huge impact on new generation experiments seals the success of the Tevatron program and paves the way for future advances in high energy physics.

\section{References}

[1] http://www-cdf.fnal.gov/physics/physics.html;

http://www-d0.fnal.gov/Run2Physics/WWW/results.htm.

[2] T. Aaltonen et al. (CDF Collaboration, DZero Collaboration), Phys. Rev. D 88, 052014 (2013).

[3] V.M. Abazov et al. (DZero Collaboration), arXiv:1407.6369, accepted by Phys. Rev. Lett.; T. Aaltonen et al. (CDF Collaboration), CDF conference note 11104; http://tevnphwg.fnal.gov/results/Higgs_JP_Summer_14/tevjp.pdf. 
[4] T. Aaltonen et al. (CDF Collaboration), Phys. Rev. Lett. 110, 121802 (2013).

[5] T. Aaltonen et al. (CDF Collaboration), CDF conference note 11079; http://wwwcdf.fnal.gov/physics/new/top/2014/wptbmetbb_webpage/cdf11079_wptbmetbb_pubnote.pdf.

[6] Tevatron Electroweak Working Group (CDF Collaboration, DZero Collaboration), arXiv:0908.2171.

[7] T. Aaltonen et al. (CDF Collaboration), Phys. Rev. Lett. 112, 231805 (2014).

[8] V.M. Abazov et al. (DZero Collaboration), Phys. Lett. B 726, 656 (2013).

[9] T. Aaltonen et al. (CDF Collaboration, DZero Collaboration), Phys. Rev. Lett. 112, 231803 (2014).

[10] T. Aaltonen et al. (CDF Collaboration), Phys. Rev. Lett. 111, 182002 (2013).

[11] V.M. Abazov et al. (DZero Collaboration), arXiv:1405.0421, accepted by Phys. Rev. D.

[12] T. Aaltonen et al. (CDF Collaboration), Phys. Rev. D 87, 092002 (2013).

[13] V.M. Abazov et al. (DZero Collaboration), DZero conference note 6445; http://wwwd0.fnal.gov/Run2Physics/WWW/results/prelim/TOP/T104/T104.pdf.

[14] T. Aaltonen et al. (CDF Collaboration), CDF conference note 11092; http://wwwcdf.fnal.gov/physics/new/top/2014/Afbbpub/cdf11092_AfbbHighMassPub.pdf.

[15] T. Aaltonen et al. (CDF Collaboration), Phys. Rev. Lett. 110, 121802 (2013).

[16] T. Aaltonen et al. (CDF Collaboration), CDF conference note 11072; http://wwwcdf.fnal.gov/physics/new/top/2014/mtop_dil/cdf11072_dil_mt_p38_pub_v1.pdf.

[17] V.M. Abazov et al. (DZero Collaboration), Phys. Rev. Lett. 113, 032002 (2014).

[18] T. Aaltonen et al. (CDF Collaboration, DZero Collaboration), arXiv:1407.2682.

[19] T. Aaltonen et al. (CDF Collaboration), CDF conference note 11098; http://wwwcdf.fnal.gov/physics/ewk/2014/WWjets/cdf11098_WW+jets.pdf.

[20] T. Aaltonen et al. (CDF Collaboration), CDF conference note 11087; http://wwwcdf.fnal.gov/physics/new/qcd/2014/vplusdstar_new/vpludstar_webpage/cdf11087_vplusdstar_public.pdf.

[21] V.M. Abazov et al. (DZero Collaboration), Phys. Rev. D 89, 072006 (2014).

[22] V.M. Abazov et al. (DZero Collaboration), Phys. Rev. Lett. 114, 042001 (2014).

[23] T. Aaltonen et al. (CDF Collaboration), Phys. Rev. D 90, 012013 (2014).

[24] V.M. Abazov et al. (DZero Collaboration), Phys. Rev. Lett. 110, 241801 (2013). 